\title{
Low-consistency refining of mechanical pulp in the light of forces on fibres
}

\author{
Jan-Erik Berg, Christer Sandberg, Birgitta A Engberg and Per Engstrand
}

KEYWORDS: Low consistency, Two-zoned refiners, Fibre length, Refining intensity, Disc gap, Specific edge load, Forces on fibres, Thermomechanical pulping

SUMMARY: The aim of this investigation was to find new approaches to evaluate the performance of lowconsistency refiners. Data from a paper mill producing TMP from Norway spruce was used in order to find a possible way to calculate the power split between the zones in a TwinFlo refiner. An assumption of equal amount of fibres captured between overlapping bars was found successful in order to develop equations for the power split. The equations predicted equal power in both zones at equal disc gaps. The power was found to increase approximately linearly with decreasing disc gap over the range, $0.1-0.2 \mathrm{~mm}$. The power split was essential to know for calculating refining intensities expressed as specific edge load and forces on fibres in the two zones. The reduction in fibre length was about $5 \%$ at $0.17 \mathrm{~mm}$ disc gap or at $0.03 \mathrm{~N}$ forces on fibres or at $0.7 \mathrm{~J} / \mathrm{m}$ specific edge load. Disc gap, forces on fibres and specific edge load was found to predict fibre shortening approximately equally upon changes in power and flow rate through the refiner.

\section{ADDRESSES OF THE AUTHORS:}

Jan-Erik Berg (jan-erik.berg@miun.se), Birgitta A Engberg (birgitta.engberg@miun.se) and Per Engstrand (per.engstrand@miun.se): FSCN, Department of Chemical Engineering, Mid Sweden University, SE-851 70 Sundsvall, Sweden Christer Sandberg (christer.sandberg@holmenpaper.com): Holmen Paper, Braviken Paper Mill, SE-601 88 Norrköping, Sweden

\section{Corresponding author: Jan-Erik Berg}

Low-consistency (LC) refining is more energy efficient compared to high-consistency (HC) refining regarding tensile strength development for mechanical pulp (Engstrand et al. 1990; Hammar et al. 1997; Sandberg, Sundström and Nilsson 2009; Hammar et al. 2010). However, LC refiners require high flow rates in relation to motor power which limit maximum possible specific energy input. Therefore the development has been towards larger multi-zone refiners and conical refiners. Today's largest two-zoned refiner, 72 inches in diameter with 5 MW motor power, is located at the Holmen Paper, Braviken Paper Mill in Norrköping, Sweden (Andersson et al. 2012a).

One complication with two-zoned refiners is that the refining conditions can differ between the zones (Andersson et al. 2012b), which mean that e.g. disc gap and power has to be measured in both zones if one wants to optimise each zone separately. Accurate measurements of both disc gaps are well established but seldom used whereas power is only measured on the refiner as a whole.
One way to determine the power split between the two zones is based upon thermodynamic calculations over the refining zones (Andersson et al. 2012b). This method requires accurate measurements of temperature and flow rate in both zones.

Fibre flexibilization is a key objective in refining. It is attained by removal of surface material from fibres, creation of new surface within fibre walls, or by softening of the wall material through mechanical stresses. The forces acting on fibres between crossing bars produce these property changes by imposing the strains that create new internal area or plastic deformation. Forces in excess of the rupture strength of fibres, however, produce fibre shortening. The expression of forces on fibres developed by Kerekes and Senger (2006) and Kerekes (2011) can be used to calculate the refining intensity.

The overall goal with this investigation was to find ways to improve understanding with regard to optimization of refining conditions in order to increase the efficiency of LC refining. If a larger share of the fibre development can be made in LC refiners instead of in $\mathrm{HC}$ refiners, the total power consumption to certain strength properties of mechanical pulps can be reduced since LC refiners are more energy efficient.

A specific objective was to develop a method to determine the power split between the two zones in order to compare the refining intensity expressed as forces on fibres with more conventional measures that give estimates of the refining intensity such as disc gap (Mohlin 2006) and specific edge load (Brecht, Siewert 1966).

\section{Materials and Methods}

The data used for this work was collected from a twozoned TwinFlo72 LC refiner from Andritz in the Holmen Paper, Braviken Paper Mill in Norrköping, Sweden (Andersson 2011). The feed pulp, characterised in Table 1, to the TF72 was TMP from Norway spruce produced in two parallel Double-Disc RGP 68DD refiners from Valmet with a specific energy consumption of around $1600 \mathrm{kWh} / \mathrm{adt}$. The TF72 refiner was fitted with 72-inch $(1.8 \mathrm{~m})$ LemaxX $^{\circledR}$ Spiral segments. The rotational speed was $320 \mathrm{rpm}$. The segment geometry is described in Table 2.

Table 1 - Characterisation of the feed pulp to the LC refiner

\begin{tabular}{|c|c|c|c|}
\hline $\begin{array}{l}\text { CSF}^{1} \\
(\mathrm{ml})\end{array}$ & $\begin{array}{l}\text { Pulp consis- } \\
\text { tency, } C_{F}(-)\end{array}$ & $\begin{array}{l}\text { Fibre length }{ }^{2,3} \\
I,(\mathrm{~mm})\end{array}$ & $\begin{array}{l}\text { Fibre width } \\
d_{0},(\mu \mathrm{m})\end{array}$ \\
\hline $97-146$ & 0.04 & $0.94-0.98$ & 27 \\
\hline
\end{tabular}

1) Canadian Standard Freeness (ISO 5267-2)

2) Length-weighted fibre length

3) From Pulp Eye ${ }^{\circledR}$ (Eurocon) 
Table 2 - Segment geometry described at radius $0.8 \mathrm{~m}$

\begin{tabular}{lllll}
\hline $\begin{array}{l}\text { Bar width } \\
W,(\mathrm{~mm})\end{array}$ & $\begin{array}{l}\text { Groove } \\
\text { width } \mathbf{G},(\mathrm{mm})\end{array}$ & $\begin{array}{l}\text { Groove } \\
\text { depth }(\mathrm{mm})\end{array}$ & $\begin{array}{l}\phi^{1} \\
\left(^{\circ}\right)\end{array}$ & $\begin{array}{l}\boldsymbol{C E}^{2} \\
(\mathrm{~km} / \mathrm{rev})\end{array}$ \\
\hline 1.65 & 3.2 & 6.6 & 27 & 414
\end{tabular}

1) Bar angle to radial direction

2) Cutting Edge Length, total for both zones

Table 3 - Refining conditions. AE represents Adjustment End and ME represents Motor End.

\begin{tabular}{lllrrl}
\hline Trial & Disc gap & Gross & \multicolumn{2}{c}{$\begin{array}{l}\text { Flow rate } \\
\text { power }\end{array}$} & \multicolumn{2}{c}{$\begin{array}{l}\text { Recirc. } \\
\text { Q(kg/s) }\end{array}$} & (\%) \\
& $\boldsymbol{T}_{\boldsymbol{R}}(\mathbf{m m})$ & power & ME & ME & \\
\hline A & $0.09-0.19$ & $2.0-2.9$ & 90 & 60 & 21 \\
B & $0.10-0.26$ & $2.0-2.9$ & 120 & 80 & 43 \\
C & $0.08-0.23$ & $2.0-3.0$ & 150 & 100 & 53 \\
\hline
\end{tabular}

The reason that the segment geometry is described at radius $0.8 \mathrm{~m}$ is to select an average radius $\langle R\rangle$ which represents the radius of average tangential velocity $\langle v\rangle$ such that the total shear force times $\langle v\rangle$ equals the power. The average radius was calculated as described by Roux et al. (2009):

$<R>=\frac{2}{3} \frac{R_{2}^{3}-R_{1}^{3}}{R_{2}^{2}-R_{1}^{2}}$

where

$R_{2}=$ outer radius (here $0.9 \mathrm{~m}$ )

$R_{1}=$ inner radius (here $0.6 \mathrm{~m}$ )

The average tangential velocity $\langle v>$ was calculated to $27 \mathrm{~m} / \mathrm{s}$ by rotational speed (rad/s) times average radius.

A schematic drawing of the DD-LC line is shown in Fig 1.

The data used here was sampled during refining trials performed in a former work reported by Luukkonen (2011). The trials (A-C) were performed at different levels of recirculation according to Table 3. Each trial was conducted with successively decreasing disc gap in order to achieve four levels of refiner motor power.

The capture of fibres by a moving bar has been studied in detail by Kerekes and Olson (2003). They found that the amount of fibres captured was a function of pulp consistency, fibre length and rotational speed. While these refining conditions are approximately constant in the present investigation it is assumed that equal amount of fibres is captured between overlapping bars for both Adjustment End (AE) and Motor End (ME).

The power split of gross power $P_{R}$ at a refiner disc gap $T_{R}$ can thus be calculated by:

$P_{X}\left(T_{R}\right)=\frac{1}{2} P_{R}\left(T_{R}\right)$

$T_{R}=\frac{1}{2}\left(T_{A E}+T_{M E}\right)$

where $X$ denotes AE or ME.

The power split at a disc gap close to $T_{R}$ i.e. $\left(T_{R}+\Delta T\right)$, where $\Delta T \ll T_{R}$, can be calculated by:

$P_{X}\left(T_{R}+\Delta T\right)=\frac{1}{2} P_{R}\left(T_{R}\right)+\frac{1}{2} \frac{\Delta P_{R}\left(T_{R}\right)}{\Delta T} \Delta T$

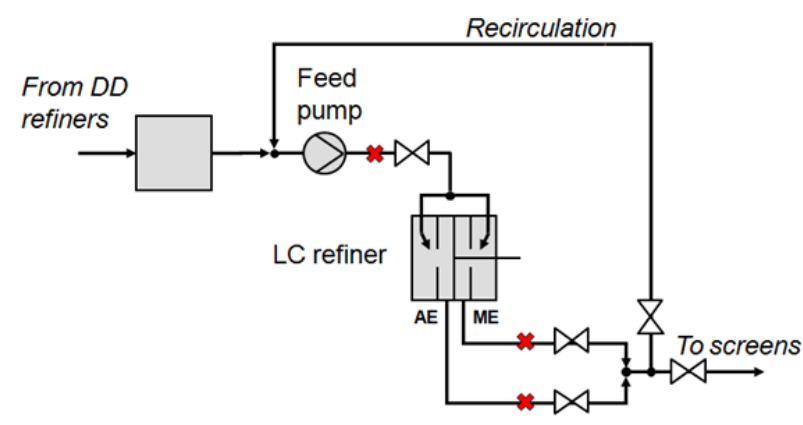

Fig 1 - Schematic drawing of the DD-LC line. Sampling points are marked with crosses. AE denotes Adjustment End and ME denotes Motor End.

The Specific Edge Load, SEL (J/m) was calculated by:

$S E L_{X}=\frac{\left(P_{X}-P_{0} / 2\right)}{\omega C E L_{X}}$

where

$P_{0}=$ No-load power (here $800 \mathrm{~kW}$ )

$\omega=$ rotational speed (here $320 / 60=5.33 \mathrm{rev} / \mathrm{s}$ )

$C E L_{X}=$ Cutting Edge Length (here $207 \mathrm{~km} / \mathrm{rev}$ )

The gross Specific Energy Consumption, SEC (J/kg) was calculated by:

$S E C_{X}=\frac{P_{X}}{Q_{X} C_{F}}$

where

$Q_{X}=$ mass flow (kg pulp suspension/s)

$C_{F}=$ pulp consistency (kg fibre/kg pulp suspension)

Average forces on fibres, $f_{N}(\mathrm{~N})$ were calculated by using the approximate expression developed by Kerekes and Senger (2006) and Kerekes (2011):

$f_{N}=\frac{\sqrt{\pi} d_{0} C_{F} l^{3 / 2} \sqrt{k G}}{4 a}\left(\frac{S E L}{\mu_{E} S g z}\right)^{1-b}$

where

$d_{0}=$ uncompressed fibre width (diameter)

$l=$ inlet fibre length

$k=$ fraction of groove width from which fibre is captured

(here 1.0 (Kerekes and Senger 2006))

$G=$ Groove width

$\mu_{E}=$ effective coefficient of friction (here set to 0.14 (Prairie et al. 2008))

$s=$ distance of bar movement over which force is exerted (here set equal to bar width, $W$ (Kerekes 2011))

$g$ = distance over bar width covered with fibres (here set equal to bar width $W$ (Kerekes 2011))

$z=$ fractional distance along unit bar length covered with

fibre (here set to 0.95 (Kerekes 2011))

$a=$ pulp mat compression parameter (here set to 0.002

(Kerekes and Senger 2006))

$b=$ pulp mat compression parameter (here set to 0.36

(Kerekes, Senger 2006)) 


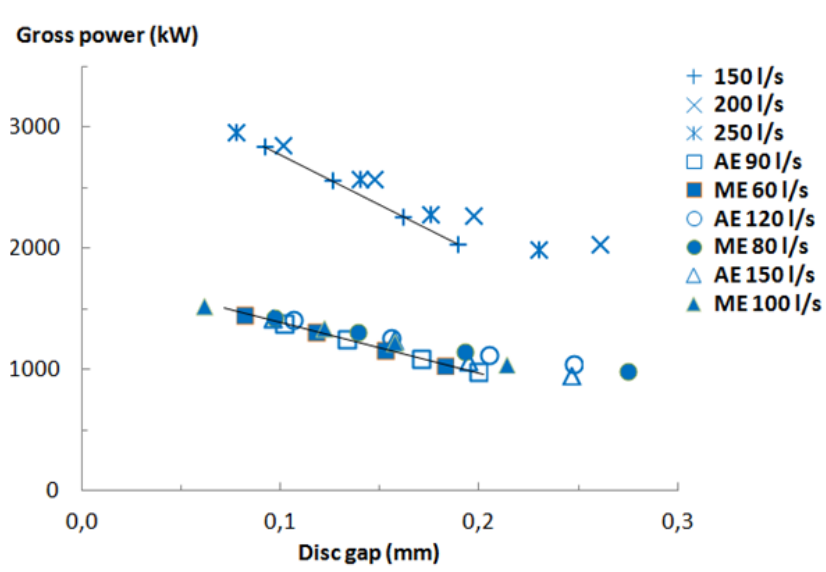

Fig 2 - Gross motor power and calculated power in Adjustment End (AE) and Motor End (ME versus disc gap at different flow rates through the refiner

\section{Results and Discussion}

Fig 2 shows the logged gross motor power (upper curve) and the calculated power in $\mathrm{AE}$ and $\mathrm{ME}$ at different flow rates using $E q$ 4. There is an approximate linear relation between power and disc gap over the range, 0.1-0.2 mm, for all tested flow rates which is demonstrated by solid lines for a total flow $150 \mathrm{l} / \mathrm{s}$ through the refiner and for 90 and $60 \mathrm{l} / \mathrm{s}$ in each zone respectively. Such a linear relation was also found by Martinez and Kerekes (1994) who carried out trails in a laboratory single bar refiner.

Lundin, Batchelor and Fardim (2008) found that a negative exponential function can describe the relation between gross power and disc gap in a laboratory conical refiner. Other researchers have found power to correlate linearly with inverse of gap size over a large range of gap size, e.g. Mohlin (2007) and Luukkonen, Olson and Martinez (2010). These functions are however approximately linear over the narrow range of gap size studied in present work.

Calculated net power in the two zones, $P_{X}-P_{0} / 2$, versus disc gap up to $0.2 \mathrm{~mm}$ is shown in Fig 3. Straight lines are fitted to the data points. The intercepts with the $\mathrm{x}$-axis can be interpreted as the critical gap $T_{c}$ where the cross section of the fibres rather than only the flocs starts to be deformed (Kerekes 2011). The critical gap $T_{c}$ was found to vary around $0.33-0.43 \mathrm{~mm}$, see Fig 3 . The difference in $T_{c}$ indicates that the floc size varied between the trials which might be explained by a change in fibre properties due to different recirculation ratios, which has been pointed out by Kerekes (2014) and possibly also by different flow conditions in the grooves. Martinez (1995) has explained how e.g. more slender fibres could result in higher values of $T_{c}$.

The split between calculated gross specific energies is shown in Fig 4. The specific energy consumption varied from about $60 \mathrm{kWh} /$ ton for flow rate $150 \mathrm{l} / \mathrm{s}$ up to $160 \mathrm{kWh} /$ ton for flow rate $60 \mathrm{l} / \mathrm{s}$ compared at disc gap $0.1 \mathrm{~mm}$.

Fig $5 a$ shows the relation between relative fibre length versus forces on fibres which is approximately linear for forces $0.01-0.05 \mathrm{~N}$. The measured outlet fibre lengths $l_{X}$

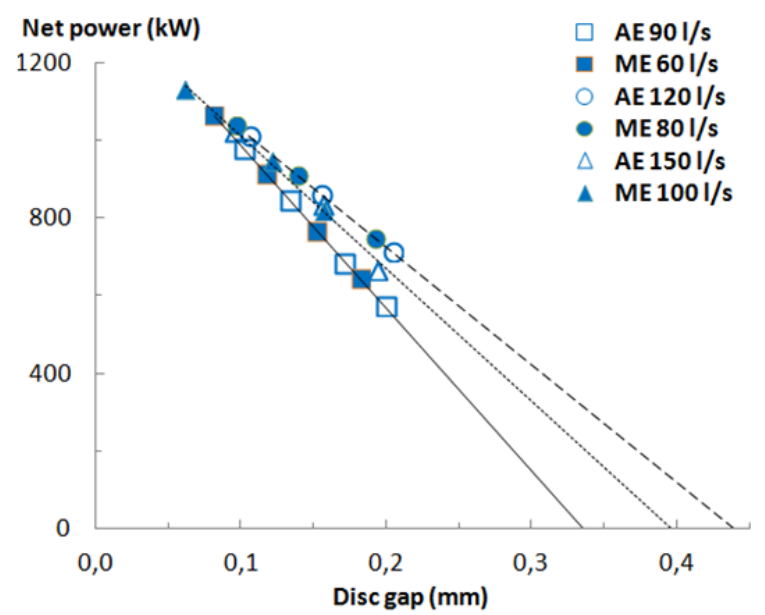

Fig 3 - Calculated net power versus disc gap for Adjustment End (AE) and Motor End (ME) at different flow rates through the refiner

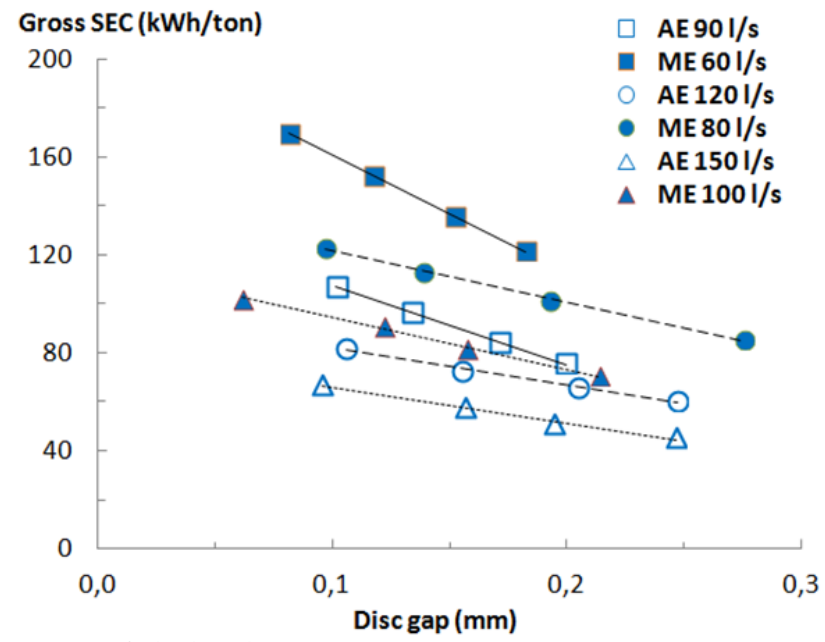

Fig 4 - Calculated gross Specific energy consumption versus disc gap for Adjustment End (AE) and Motor End (ME) at different flow rates through the refiner

are normalised to the inlet fibre length $l$. The force corresponding to, e.g. 0.95 relative fibre length can be estimated to about $0.03 \mathrm{~N}$. Fig $5 a$ shows that it is possible to use forces on fibres to predict fibre shortening for the refining conditions studied.

Other measures for refining intensity are disc gap and Specific Edge Load (SEL). Fig $5 b$ shows relative fibre length versus disc gap. The gap corresponding to 0.95 relative fibre length can be estimated to $0.17 \mathrm{~mm}$ and Fig $5 c$ shows that the SEL corresponding to 0.95 relative fibre length can be estimated to $0.7 \mathrm{~J} / \mathrm{m}$. The three different expressions for refining intensities shown in Fig $5 a-c$ give the impression to predict fibre shortening approximately equally upon changes in power and flow rate through the refiner.

Even though the gross specific energies were varied in a wide range by using different flow rates and disc gaps, shown in Fig 4, the fibre length seems to be affected only by the refining intensities as shown in Fig 5a-c. 

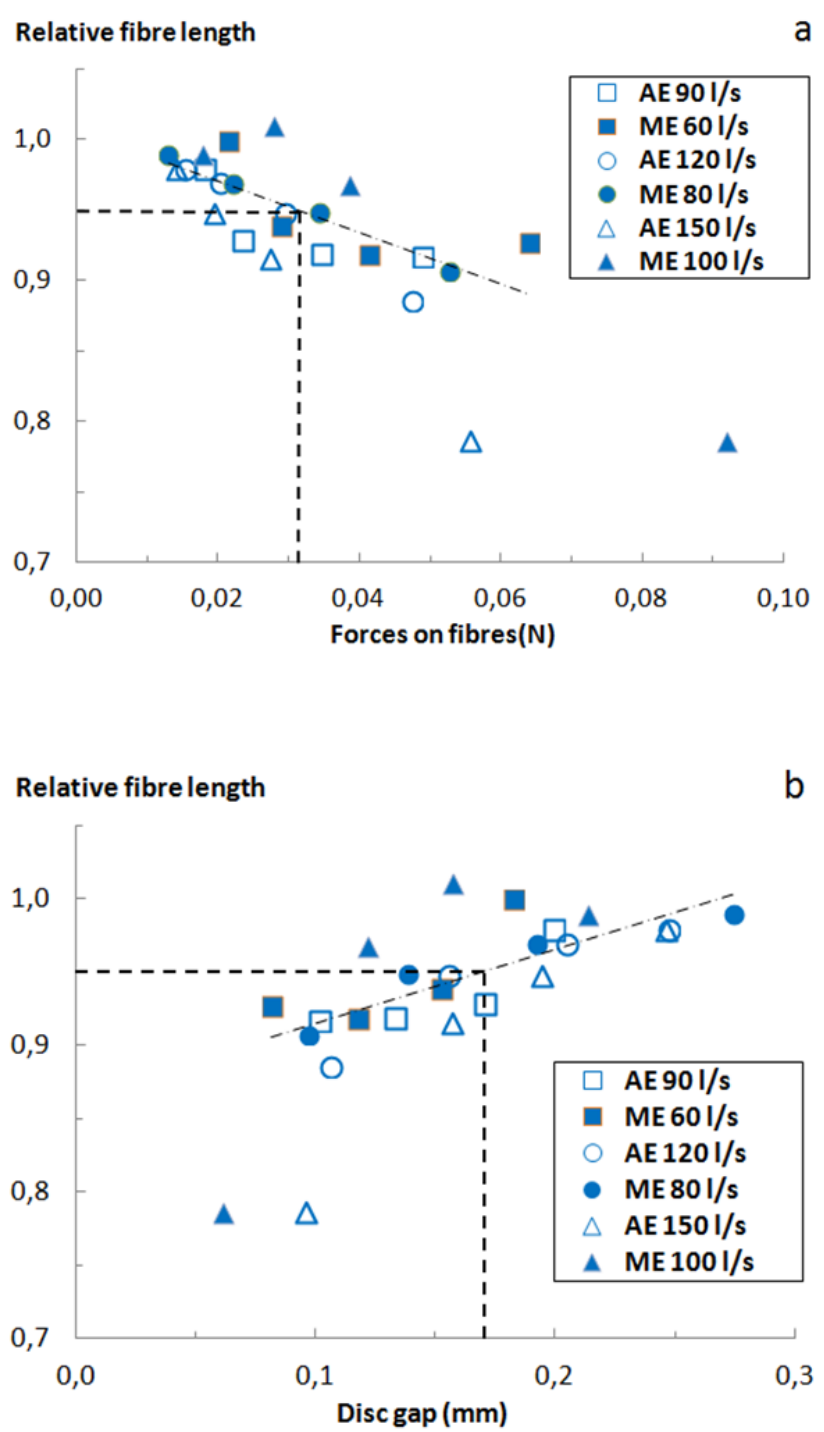

Relative fibre length

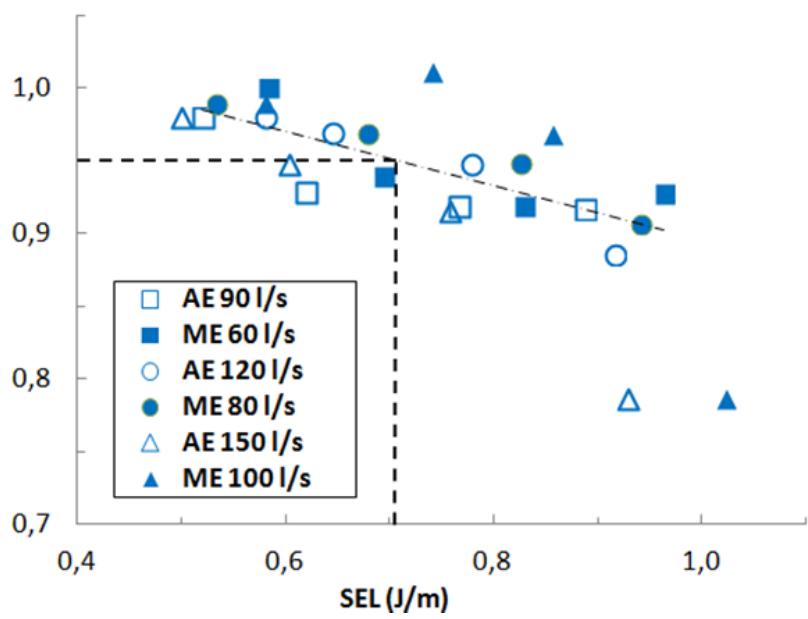

Fig 5 - Relative fibre length (IX/l) versus (a) Forces on fibres, (b) Disc gap and (c) Specific Edge Load (SEL) for Adjustment End $(\mathrm{AE})$ and Motor End (ME) at different flow rates through the refiner. Values corresponding to 0.95 relative fibre length are indicated.
For a specific refiner operating on a reasonably constant feed pulp it is thus good enough to use the rather simple parameter specific edge load to control the refining intensity. If the feed pulp changes, the situation is not so simple, though.

The fibre length reduction can be very different for a specific refiner setup (model, segment design, power, flows, etc.) as was shown in earlier work by Sandberg, Sundström and Nilsson (2009). Changing segment design will of course also affect the process control parameters.

Given the heterogeneity of the refining process, e.g. force distribution on flocs, there will always be some cutting when forces are large enough on average to refine the bulk of the fibres. From Fig 5b, drastic fibre cutting takes place at around gap size $0.1 \mathrm{~mm}$. This corresponds to a force of about $0.06 \mathrm{~N}$ in Fig $5 a$, which, remarkably, is exactly the rupture strength of undamaged native softwood fibres (Burgert et al. 2002).

A TMP fibre is however normally weaker compared to a native fibre due to e.g. split fibres and that parts of the cell wall layers have been peeled off (Reme et al. 1999). Somboon and Paulapuro (2009) have shown that fibres in TMP that is refined from 600 to $100 \mathrm{ml}$ CSF lose approximately $30-40 \%$ of rupture strength.

\section{Conclusions}

An assumption of equal amount of fibres captured between overlapping bars in the two refining zones was found successful in order to develop equations for the power split in a two-zoned LC refiner. The equations predicted equal power in both zones at equal disc gaps, and the power was found to increase approximately linearly with decreasing disc gap over the range, 0.1$0.2 \mathrm{~mm}$. The calculated power split was essential for calculating specific edge load and forces on fibres in the two zones.

The average forces on fibres were about $0.03 \mathrm{~N}$ at 0.95 relative fibre length. The corresponding disc gap and specific edge load were calculated to $0.17 \mathrm{~mm}$ and $0.7 \mathrm{~J} / \mathrm{m}$ respectively. Disc gap, forces on fibres and specific edge load were found to predict fibre shortening approximately equally upon changes in power and flow rate through the refiner.

For a specific refiner operating on a reasonably constant feed pulp it is thus good enough to use the rather simple parameter specific edge load to control the refining intensity.

\section{Acknowledgements}

The authors are most grateful to the Knowledge Foundation for financial support to Energy Efficient Mechanical Pulping Research Profile, e2mp-rp and Holmen Paper, Braviken Paper Mill for providing data.

Literature

Andersson, S. (2011): Low consistency refining of mechanical pulp - process conditions and energy efficiency, Licentiate thesis, Mid-Sweden University, Sundsvall, Sweden.

Andersson, S., Sandberg, C. and Engstrand, P. (2012a): Comparison of mechanical pulps from two stage $\mathrm{HC}$ single disc and $\mathrm{HC}$ double disc-LC refining, Appita J. 65(1), 57-62. 
Andersson, S., Sandberg, C. and Engstrand, P. (2012b): The effect of rotor position on pulp properties in a two-zoned low consistency refiner, Nordic Pulp Paper Res. J. 27(3), 525-530.

Brecht W. and Siewert W. (1966): Zur theoretisch-technischen Beurteilung des Mahlprozesses modern Mahlmaschinen, Das Papier 20(1), 4-14.

Burgert, I., Keckes, J., Frühmann, K., Fratzl, P. S. and Tschegg, E. (2002): A comparison of two techniques for wood fibre isolation - evaluation by tensile tests on single fibres with different microfibril angle, Plant Biol. 4(1), 9-12.

Engstrand, P. O., Hammar, L. Å., Htun, M. T., Pettersson, R. L. and Svensson, B.N. (1990): Framställning av mekanisk massa i två steg (Two-step manufacture of mechanical pulp), Swedish patent 8801731-4, 461103.

Hammar, L. A., Htun, M. and Svensson, B. (1997): A twostage refining process to save energy for mechanical pulps, Int. Mech. Pulping Conf., Stockholm, Sweden, pp. 257-262.

Hammar, L. Å., Salmén, L., Sandberg, C. and Sundström, L. (2010): The Effect of Process Conditions on Pulp Quality Development in Low Consistency Refining of Mechanical PulpTMP, Appita J. 63(5), 377.

Kerekes, R. J. (2011): Force-based characterization of refining intensity, Nord. Pulp Paper Res. J. 26(1), 14-20.

Kerekes, R. J. (2014): A theoretical analysis of recirculation in pulp refiners, TAPPI J. 13(4), 29-32.

Kerekes, R. J. and Olson, J. A. (2003): Perspectives on Fibre Length Reduction in Refining, PIRA International Conference on Scientific and Technical Advances in Refining and Mechanical Pulping, Paper 2, Stockholm, Sweden, (2003).

Kerekes, R. J. and Senger, J. J. (2006): Characterizing refining action in low-consistency refiners by forces on fibres, $\mathrm{J}$. Pulp Paper Sci. 32(1), 1-8.

Lundin, T., Batchelor, W. and Fardim, P. (2008): Fiber trapping in low-consistency refining: New parameters to describe the refining process, TAPPI J. 7(7), 15-21.

Luukkonen, A. (2011): Development of a methodology to optimize low consistency refining of mechanical pulp, Doctoral thesis, University of British Columbia, Vancouver, Canada.
Luukkonen, A., Olson, J. A., and Martinez, D. M. (2010): Low Consistency Refining of Mechanical Pulp, Effect of Gap, Speed and Power, J. Pulp Paper Sci. 36(1), 28-35.

Martinez, M. (1995): The energy expended on pulp fibres during low consistency refining, Doctoral thesis, University of British Columbia, Vancouver, Canada.

Martinez, D. M. and Kerekes, R. J. (1994): Forces on fibers in low-consistency refining, TAPPI J. 77 (12), 119-123.

Mohlin, U.-B. (2006): Refining intensity and gap clearance, 9th Pira Int. Refining, Conf., Vienna, Austria.

Mohlin, U.-B. (2007): Refiner response: gap clearance - power relationship and effect of fibre properties, In: Refining and Mechanical Pulping, Pira Int., Leatherhead, UK.

Prairie, B., Wild, P., Byrnes, P., Olender, D., Francis, W. and Ouellet, D. (2008): Forces during Bar-Passing Events in LowConsistency Refining: Distributions and Relationships to Specific Edge Load, J. Pulp Paper Sci. 34(1), 1-8.

Reme, P. A., Johnsen, P. O. and Helle, T. (1999): Changes induced in early-and latewood fibres by mechanical pulp refining, Nordic Pulp Paper Res. J., 14(3), 256-262.

Roux, J. C., Bloch, J. F., Bordin, R. and Nortier, P. (2009): The Net Normal Force Per Crossing Point: A Unified Concept for the Low Consistency Refining of Pulp Suspensions, 14th Fundamental Research Symposium: Advances in Pulp and Paper Research, FRC, Oxford, UK, pp. 51-83.

Sandberg, C., Sundström, L. and Nilsson, L. (2009): Potential of low consistency refining of TMP - mill evaluation, Int. Mech. Pulping Conf., Sundsvall, Sweden, pp. 186-189.

Somboon, P. and Paulapuro, H. (2009): Determination of wet fiber strength. Tappi J. 8(5), 4-7.

Manuscript received September 18, 2014 Accepted February 16, 2015 